\title{
No effect of inhibitors of fatty acid synthesis on profile of fatty acids synthesized by mammary homogenate*
}

\author{
T.C. Wright ${ }^{1,3}$, J.T. Brenna ${ }^{2}$, J.P. Cant ${ }^{1}$ and B.W. McBride ${ }^{1}$ \\ ${ }^{I}$ Department of Animal and Poultry Science, University of Guelph \\ Guelph, ON, Canada N1G 2W1 \\ ${ }^{2}$ Division of Nutritional Sciences, Cornell University \\ Ithaca, NY, USA, 14853
}

\begin{abstract}
In vitro incubations using bovine mammary post-mitochondrial supernatant were conducted using a stable isotope of acetate to measure de novo fatty acid synthesis. Incubations were done with and without enzyme inhibitors for acetyl CoA carboxylase and fatty acid synthase to determine the effects of partial inhibition on total enzyme activity on the fatty acids synthesized. There was no significant change in composition of fatty acids synthesized and the results of this experiment confirm that the use of enzyme inhibition in this pathway is suitable for experiments to apply metabolic control analysis.
\end{abstract}

KEY WORDS: mammary, fatty acids, in vitro, carboxylase, control analysis

\section{INTRODUCTION}

Metabolism in biochemical pathways has often been qualitatively described in terms of the individual steps within the pathway, rather than looking at the pathway as a whole. A more useful approach that increases our understanding of the control and regulation of biological pathways is described by metabolic control analysis (MCA). Metabolic control analysis was originally described by Kacser and Burns (1973). This approach evaluates control within a philosophy of shared or distributed control, among the individual elements of the pathway of interest. The alternative concept of single control points, often described as rate-

\footnotetext{
* Supported by NSERC Canada and technical assistance of Peter Lawrence (Cornell University) was appreciated for this study

${ }^{3}$ Corresponding author: e-mail: tcwright@uoguelph.ca
} 
limiting or pace-maker steps is replaced by the determination of control coefficients that estimate the proportion of control exerted by individual steps. Acetyl CoA carboxylase (ACC; EC 6.4.1.2) is often described as rate-limiting for de novo fatty acid synthesis. To determine the degree of control of a single enzyme over flux through the entire pathway, the enzyme activity is manipulated using activators or inhibitors. The objective of this experiment was to determine if the pattern of fatty acids synthesized in mammary homogenates was significantly affected by inhibition of ACC and fatty acid synthase (FAS; EC 2.3.1.85).

\section{MATERIAL AND METHODS}

The in vitro incubations used to synthesize fatty acids from mammary tissue were adapted from the methods of Wright et al. (2002). Modifications to the methodology included the addition of $5.0 \mu \mathrm{L}$ of protease inhibitor (Sigma Aldrich P8340, Oakville, ON), desalting the post-mitochondrial mammary supernatant by using a centrifugal concentrator containing a 10k molecular weight filter (Nanosep, Northborough, MA), and the replacement of radiolabelled acetate with $\left[1,2-{ }^{13} \mathrm{C}\right]$ acetate (Cambridge Isotope Laboratories Inc., Andover, MA). A series of preliminary incubations were conducted to determine a satisfactory isotopic dilution rate. Based on those results, stable isotope was diluted 1:50 with natural abundance acetate. Control incubations without enzyme inhibitors and incubations with avidin and pyridoxal 5-phosphate were conducted to partially inhibit total ACC and FAS activity, respectively. After $60 \mathrm{~min}$ the incubation tubes were immediately frozen. Fatty acids were extracted and fatty acid methyl esters were prepared. Isotopic enrichment of the fatty acids was measured using high precision isotope ratio mass spectrometry according to the methods of Su and Brenna (1998).

\section{RESULTS}

Acetate incorporation into fatty acids from $\mathrm{C} 10: 0$ to $\mathrm{C} 18: 1$ is shown in Figure 1. Peak incorporation of acetate was into C14:0 for inhibited and control incubations, which indicates that there is a difference between the in vitro synthesis of fatty acids using this preparation and the in vivo synthesis of fatty acids in bovine mammary tissue, where C16:0 is the major product. There was no statistical difference from the control for fatty acids synthesized with the low concentrations of inhibitor used herein except for higher $(\mathrm{P}<0.05)$ acetate incorporation for $\mathrm{C} 18: 0$ for the pyridoxal 5-phosphate and 1- $\mu \mathrm{M}$ avidin treatments. Acetate incorporation into $\mathrm{C} 18: 0$ and $\mathrm{C} 18: 1$ was, however, lower than the incorporation into the other fatty acid pools. Numerical reductions from the control incubations with a higher concentration of avidin may indicate the effect of the stronger inhibitor concentration. The inhibitors may affect short chain fatty 
acids (not separated), which are a significant product of mammary fatty acid synthesis. The change in the pattern of fatty acids synthesized was examined by standardizing the fatty acids relative to the production of C10:0 (Figure 2), which demonstrates the similarity in fatty acids synthesized under the control conditions and in the presence of inhibitors.

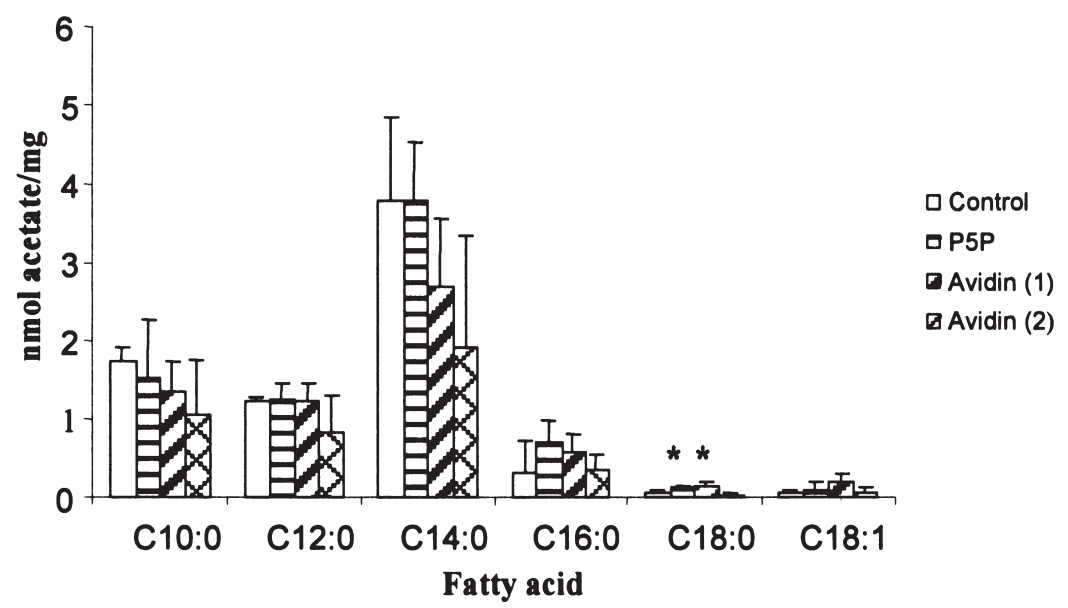

Figure 1. Acetate incorporation into fatty acids from control and inhibited incubations ( $\mathrm{P} 5 \mathrm{P}=$ pyridoxal 5-phosphate, and avidin at 1 and $2 \mu \mathrm{M})$. *denotes statistical difference from control $(\mathrm{P}<0.05)$

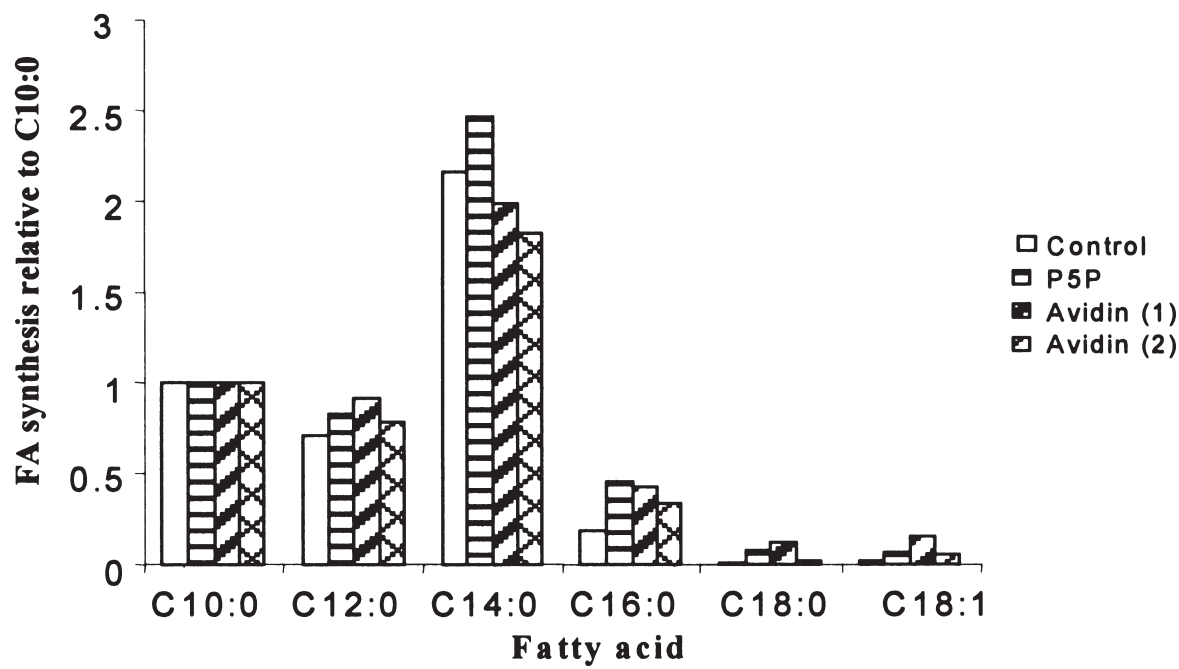

Figure 2. Synthesis of fatty acids relative to the production of $\mathrm{C} 10: 0$ from control and inhibited incubations $(\mathrm{P} 5 \mathrm{P}=$ pyridoxal 5-phosphate, and avidin at 1 and $2 \mu \mathrm{M})$ 


\section{DISCUSSION}

The use of partial enzyme inhibition to determine control coefficients for ACC and FAS requires that the end products of fatty acids synthesis are consistent, or the application of metabolic control analysis is confounded by the changes to the products synthesized by the pathway. The predominant end product, namely C14:0 rather than C16:0 suggests differences in chain termination compared to in vivo synthesis. The present incubation system results in the synthesis of free fatty acids rather than triacylglycerols (Wright et al., 2002). The results reported herein indicate that partial enzyme inhibition is appropriate to alter the overall pathway flux without significantly changing the composition of fatty acids produced. Acetyl-CoA carboxylase remains a primary enzyme of interest in fatty acid biosynthesis. Davis et al. (2000) examined the effects of ACC overexpression in $E$. coli and reported a six-fold increase in fatty acid synthesis, but with a concomitant increase in malonyl-CoA, which suggests that there could be additional control points, including FAS, to regulate fatty acid biosynthesis.

\section{CONCLUSIONS}

The synthesis of de novo fatty acids from bovine mammary homogenate resulted in the same relative composition of fatty acids produced whether inhibitors of ACC or FAS were added independently or not. The fractional enzyme inhibition technique of MCA is therefore appropriate for this pathway.

\section{REFERENCES}

Davis M.S., Solbiati J., Cronan Jr. J.E., 2000. Overproduction of acetyl-CoA carboxylase activity increases the rate of fatty acid biosynthesis in Escherichia coli. J. Biol. Chem. 275, 2859328598

Kacser H., Burns J.A., 1973. The control of flux. Symp. Soc. Exp. Biol. 28, 65-104

Su H.-M., Brenna J.T., 1998. Simultaneous measurement of desaturase activities using stable isotope tracers or a nontracer method. Anal. Biochem. 261, 43-50

Wright T.C., Cant J.P., McBride B.W., 2002. Inhibition of fatty acid synthesis in bovine mammary homogenate by palmitic acid is not a detergent effect. J. Dairy Sci. 85, 642-647 